\title{
Les professeurs de français à Palerme de 1860 à 1900
}

Jacqueline Lillo

\section{(2) OpenEdition Journals}

Electronic version

URL: https://journals.openedition.org/dhfles/3073

DOI: $10.4000 /$ dhfles.3073

ISSN: 2221-4038

\section{Publisher}

Société Internationale pour l'Histoire du Français Langue Étrangère ou Seconde

\section{Printed version}

Date of publication: 1 June 1999

Number of pages: p.453-480

ISSN: 0992-7654

\section{Electronic reference}

Jacqueline Lillo, "Les professeurs de français à Palerme de 1860 à 1900", Documents pour I'histoire du français langue étrangère ou seconde [Online], 23 | 1999, Online since 28 August 2015, connection on 10 March 2023. URL: http://journals.openedition.org/dhfles/3073; DOI: https://doi.org/10.4000/dhfles. 3073

This text was automatically generated on 10 March 2023.

All rights reserved 


\title{
Les professeurs de français à Palerme de 1860 à 1900
}

\author{
Jacqueline Lillo
}

1 Le présent article, résultat d'un travail de compilation d'archives, se propose de donner une suite à Enseignants et élèves de français à Palerme de 1800 à 1860 (Lillo, 1992), publié dans le numéro spécial sur « Maîtres et élèves » coordonné par Herbert Christ et Caria Pellandra.

2 En 1861, Palerme est une ville de 194436 habitants. Depuis l'unification (1860) elle a perdu l'importance politique qu'elle avait précédemment lorsqu'elle était siège du vicerègne des Deux-Siciles (jusqu'en 1812) puis de la Luogotenenza.

3 Mis à part les familles aristocratiques qui s'appauvrissaient lentement et dignement et les quelques riches bourgeois ou nouveaux financiers qui avaient fait fortune, la majorité de la population était tellement pauvre que, dans les années 80, la municipalité fut obligée de consacrer une bonne partie de ses revenus en bienfaisance et que le problème de la distribution du blé et le prix du pain restait crucial pour toute cette fin de siècle.

4 Les conditions hygiéniques étaient des plus précaires, le réseau souterrain des égouts presque inexistant, aussi les épidémies se multiplièrent-elles jusqu'à la fin du siècle. Lors des épidémies de choléra en 1867, 1885--1886, 1887, 1893, 1896-1897, les écoles furent fermées et les élèves renvoyés chez eux. De fausses rumeurs (on craignit même une épidémie de peste bubonique dans les années 90) provoquèrent des psychoses et l'abandon des villes par les professeurs et les élèves.

5 Du point de vue politique les temps étaient troublés. Le gouvernement piémontais avait peur des conspirations et des émeutes fomentées aussi bien par les socialistes que par les légitimistes. Il en résulta une mainmise policière extrêmement lourde sur la population. L'état centralisateur (sur le modèle de la France) et autoritaire voulait tout régenter et tout savoir. Ainsi, c'était le ministère qui octroyait toute autorisation d'absence ou de congés et non pas le directeur de l'école ou le provveditore (recteur de l'Académie). En 1862, un inspecteur ministériel s'introduisit pendant la nuit dans l'école technique de Palerme (la seule existante à l'époque) pour contrôler les papiers 
du directeur et les registres des professeurs. Il est vrai que le peuple sicilien étant, par nature, " désobéissant, séditieux et indiscipliné ", comme le décrivait au siècle précédent Caracciolo, vice-roi des Deux-Siciles (Cancila, $1988: 6$ ), les instructions (lois, décrets, circulaires) en provenance du gouvernement central restaient le plus souvent lettre morte. La capacité de résistance passive des Siciliens dans ce domaine est d'ailleurs, aujourd'hui encore, notoire.

De 1860 à 1880, le gouvernement procéda à l'expropriation et à la vente des biens ecclésiastiques dans le but d'en utiliser les revenus pour améliorer l'éducation nationale ${ }^{1}$ qui était dans un état d'abandon désastreux. En fait, le taux d'analphabétisme en 1861 était épouvantable en Sicile $(88,88 \%)$ même si Palerme était la province la plus « instruite » (85,06 \% d'analphabètes contre $91,72 \%$ à Agrigente par exemple). Le 15 juillet 1877 une loi rendait finalement obligatoire l'enseignement primaire du premier degré. En 1901, le taux en Sicile n'était plus que de $70,89 \%$ et 62,49 \% à Palerme (Costa, $1990: 109)$ ce qui indiquait une amélioration notoire due à l'action résolue du gouvernement.

7 Dans cette société extrêmement hiérarchisée, où il était très difficile de passer d'une couche sociale à une autre, les familles aisées envoyaient leurs enfants dans les « gymnases $\|^{2}$ et lycées. Ces établissements ne programmaient pas l'enseignement des langues modernes (ou très peu pendant la période considérée puisque l'on trouve un professeur de français seulement au « Lycée-gymnase national » en 1860 et au lycée Umberto, en 1880-81, et il s'agissait alors d'un cours facultatif). En fait, ce ne fut qu'en 1889 que le français devint officiellement facultatif dans les " gymnases ", puis obligatoire en 1892. A partir de 1898, cet enseignement se poursuivit dans les classes du lycée. Les enfants de la petite bourgeoisie, des artisans et employés, quand ils ne se contentaient pas des classes du primaire, suivaient, dans le meilleur des cas, les cours des écoles et instituts techniques ${ }^{3}$ qui, au contraire, proposaient dès le début l'étude du français dans un but pratique ${ }^{4}$. Pour ces jeunes, l'objectif était de gagner leur pain le plus rapidement possible afin de pouvoir aider leurs familles 5 . Le français était aussi enseigné au Collège Nautique, au Collège San Rocco, au Collège de musique du «Buon Pastore » et dans certains établissements privés.

Il s'en suivit une modification du statut du français qui passait ainsi de langue de culture (avant 1860) à langue de communication (même si, comme nous le verrons bientôt, les résultats furent bien décevants). Ce déclassement du français se fit non pas tellement par rapport à d'autres langues étrangères (même si l'allemand, et l'anglais notamment, étaient de plus en plus étudiés), mais surtout par rapport à l'italien. Après l'unification politique, le gouvernement des Savoie voulut aussi réaliser l'unification linguistique au détriment des patois locaux et, si nécessaire, des langues étrangères.

9 Dans l'ensemble, l'éducation des filles était peu prise en compte et il fallut attendre 1899 pour voir s'ouvrir la première école technique pour jeunes filles (quelques années auparavant, cependant, une section féminine avait été ouverte dans l'Ecole technique Gaggini).

10 Les documents d'archives révèlent deux scandales qui ont déterminé l'envoi systématique, à partir des années 1880 , de tous les documents au ministère au lieu de les conserver à Palerme. Le premier est relatif au directeur de l'Ecole technique Piazzi, qui vendait les promotions et les diplômes de licenza, faisait pression sur les enseignants pour obtenir des cadeaux personnels, organisait des loteries à l'intérieur de l'école à son profit, et fut même responsable de la mort, à la suite d'une fausse-couche (1881), 
d'une jeune enseignante " continentale ${ }^{6}$ ". L'autre scandale concerne le recteur de l'Académie Baggiolini qui vendait, lui aussi, promotions et diplômes et légiférait en maître absolu. L'inspecteur ministériel envoyé pour contrôler sa gestion fut au comble de la stupeur quand il se rendit compte qu'un même procès-verbal de séances se répétait à l'infini pour toutes les réunions (seule la date différait) et que les archives de l'Académie étaient extrêmement désordonnées, souvent même inexistantes.

\section{Les écoles}

Le nouveau gouvernement, ayant trouvé l'éducation nationale dans un état désastreux, décida de tout réorganiser (loi Casati) afin de former une jeunesse reconnaissante, disciplinée et loyale. Bien qu'il ait donné une plus grande impulsion au développement des « lycées-gymnases » et des écoles normales, il n'en a pas moins créé un certain nombre d'écoles et d'instituts techniques qui, de toute façon, vu la quantité des inscriptions, étaient toujours insuffisants : la Regia Scuola Tecnica Principale qui devint : la Regia Scuola Tecnica Gaggini, la Regia Scuola Tecnica bis rebaptisée Regia Scuola Tecnica Piazzi et la Regia Scuola Tecnica D'Acquisto, enfin Ylstîuto tecnico appelé par la suite Filippo Parlatore.

De nouveaux établissements privés furent créés tel le Randazzo ; d'autres, privés ou communaux, ont survécu aux bouleversements de 1860 et ont continué d'opérer dans la Palerme post-unitaire tels le Collège San Rocco, l'Epicarme, le Veneziano pour les garçons, le collège de Maria Giusino ou le Maria Adélaïde pour les filles. Cependant nombre d'écoles ne sortirent pas indemnes de la révolution politique. Les désordres ayant entraîné la désertion des élèves, elles durent fermer leurs portes pour cause de débâcle financière.

Dans l'ensemble, les locaux étaient souvent délabrés, quelques murs mêmes croulaient de temps en temps, ce qui entraînait évidemment l'interruption des cours parfois jusqu'à trois mois (1885 à l'Ecole Gaggini, 1885-1886 à l'Institut Parlatore). D'autre part, comme le révèlent les rapports d'inspection, les écoles étaient le plus souvent dépourvues de cour de récréation, de cabinet de sciences, de bibliothèque, de salle de gymnastique. Quant aux sanitaires...

\section{Les classes, les élèves}

14 La politique suivie par le gouvernement provoqua un boom de la population scolarisée. Or comme les infrastructures et les subventions allouées étaient insuffisantes, les classes furent pléthoriques jusqu'à la fin du siècle. Cinquante, soixante élèves par classe représentaient la norme et il y eut même des pointes de soixante-dix, quatre-vingts ! En 1872, par exemple, dans la seconde de l'Ecole technique bis (en attente d'être baptisée), on pouvait compter un " nombre extraordinaire d'élèves » : 79 en tout (P. CPS 3) ! En 1876 on n'arrivait pas encore à accepter dans les écoles techniques de la ville tous les élèves qui voulaient s'inscrire ${ }^{7}$. En $1885, l^{1}$ 'Institut technique Filipo Parlatore est le plus peuplé du royaume : 422 élèves pour 8 classes, soit une moyenne de plus de cinquante par classe!

15 Tous les proviseurs cependant se plaignaient d'absences continuelles et d'abandons innombrables en cours d'année. Les raisons en étaient multiples, mais les plus 
fréquentes étaient d'ordre économique. Ainsi les familles nombreuses, presque toutes avec un seul revenu, ne pouvaient pas payer les frais d'inscription à l'école ou aux examens qui sanctionnaient chaque année de cours ${ }^{8}$. Elles retiraient donc leurs enfants de façon momentanée (ce qui entrânait souvent un redoublement) ou définitive. D'autre part, les travaux des champs : vendanges et récolte des olives à l'automne, moissons en été, rappelaient vers les campagnes ces jeunes qui apportaient ainsi une aide, même si elle était minime, au budget de la famille. L'indifférence des familles à la scolarisation des enfants était d'ailleurs une donnée constante et nombreux étaient les pères de famille qui considéraient le temps passé à l'école comme du temps perdu.

En 1830 le taux de mortalité avant l'âge de cinq ans est de 461 pour 1000 (Cancila, 1988

: 52) ! Vu les conditions d'insalubrité de quartiers entiers de la ville, les élèves s'absentaient souvent à cause de maladies. Certains même décédaient en cours de scolarité9.

D'autre part, tout événement, important ou non, était une bonne excuse pour s'absenter : les anniversaires et les fêtes du roi, de la reine, les naissances et deuils royaux (et même de certaines autres familles régnantes), etc. En 1882, les Palermitains ont en plus fêté le sixième centenaire des Vêpres Siciliennes (1282), le séjour de Garibaldi dans leur ville et l'Exposition Nationale qui s'y déroulait. Enfin, l'habitude de donner, pendant un certain temps, à midi, une récréation d'une heure, incitait les jeunes, poussés par la faim, à rentrer chez eux.

18 L'indiscipline incontrôlable était une autre donnée sans cesse commentée par tous les inspecteurs et proviseurs. Tout y contribuait, les classes pléthoriques, le manque de ponctualité systématique des jeunes, les attitudes violentes et mafieuses de certains élèves qui portaient armes ou bâtons en classe. D'autre part nombreux étaient les élèves sans livres parce que leur famille était trop pauvre pour pouvoir les leur acheter. Enfin les différences d'âge à l'intérieur d'une même classe étaient souvent excessives ; ainsi pendant l'année scolaire 1874-1875, dans une classe de troisième de l'Ecole technique bis l'écart entre le plus jeune et le plus âgé était de six ans ! Comme conséquence le nombre de jours de renvoi était impressionnant : 518 jours d'expulsion temporaire de l'école technique d'Acquisto pendant l'année scolaire 1892-1893 sur un total de 247 élèves.

19 Il est donc évident, dans ces conditions, que les déclarations d'intention de la part des professeurs restaient souvent lettre morte, que les instructions et programmes officiels étaient ignorés et que les résultats étaient pour ainsi dire nuls. Le pourcentage de réussite était souvent très bas. Malgré cela, certains inspecteurs se plaignaient que les professeurs étaient excessivement indulgents aux examens pour ne pas mettre en évidence les lacunes de leur enseignement.

\section{Les professeurs}

20 Les professeurs pouvaient être soit titulaires, soit " reggente " quand manquait le titulaire (ces derniers étaient alors le plus souvent nommés pour trois ans renouvelables), soit « incaricati », c'est-à-dire chargés annuellement de cours.

21 Ils étaient tous soumis à la toute puissance du ministère qui demandait aux proviseurs deux rapports par an. Ceux-ci envoyaient leur jugement sur le caractère des personnes, sur la qualité de l'enseignement et les résultats obtenus. Ils n'hésitaient pas à conseiller 
des mutations qu'ils justifiaient le plus souvent par l'incapacité de la part de l'intéressé de faire respecter la discipline en classe. Dans l'ensemble, cependant, les enseignants demandaient à rester à Palerme et priaient le directeur d'intercéder dans ce sens auprès du ministère.

Nous avons vu que, jusqu'en 1860, la majorité des enseignants dans les institutions privées et publiques était francophone (Lillo, 1992 : 14), avec un bon nombre de dames françaises qui tenaient des maisons d'éducation. Après l'unification, sauf exception, le professeur devait être de nationalité italienne et prêter serment de fidélité au roi. Il jurait solennellement devant le Préfet, ou un fonctionnaire qui le représentait, en présence de deux témoins, à genoux et la main sur les saintes écritures. La formule était la suivante : Moi, (nom, prénom), je jure d'être fidèle au Roi et à ses successeurs royaux et d'observer fidèlement le statut et les lois de l'état et d'exercer mes fonctions pour le seul bien inséparable du Roi et de la patrie ${ }^{10}$.

Ils devaient présenter un certificat de bonne conduite morale et politique délivré par la préfecture. Au fur et à mesure que passèrent les années, le gouvernement se préoccupa beaucoup plus des socialistes républicains que des légitimistes.

Ces enseignants ne suivaient aucune formation, mais la patente était en principe obligatoire pour pouvoir enseigner. On pouvait l'obtenir sur seule présentation des titres (mais après 1860 les jurys semblent être de plus en plus exigeants), en passant des concours (libres, réservés ou sur titres) et aussi... par simple piston. Malgré cela, comme nous le verrons (tableau 1), une bonne partie des enseignants étaient dépourvus de patente.

Ils enseignaient le plus souvent deux matières ou, de préférence, deux langues (français/allemand, etc.). Les articles 259 et 292 de la loi fondamentale prévoyaient 15 heures d'enseignement par semaine alors que les professeurs de mathématiques ou d'italien n'en faisaient que 11 "parce qu'ils (avaient) des devoirs à corriger». Les heures supplémentaires n'étaient pas rétribuées.

Les carrières étaient extrêmement lentes et les promotions difficiles à obtenir. Ainsi, par exemple, Carlo Oswald, qui vécut en Suisse, se présenta au concours pour un poste d'enseignant de français et d'allemand en 1865, Il obtint la patente en 1866. Déjà suppléant en 1865 dans lesécoles techniques de la ville (Lillo, 1993 : 28), il le sera encore en 1896, à l'âge de54 ans, à l'Ecole technique d'Acquisto malgré l'excellent jugementdu directeur ${ }^{11}$.

En fait le chômage existait déjà à Palerme pour les professeurs defrançais. On a pu trouver dans les archives de nombreuses lettres en provenance de différentes régions d'Italie qui demandaient un poste, même enprovince. Nous n'avons trouvé aucun cas de réponse positive. D'ailleurs même les gagnants des concours locaux restaient sans travail (par exemple Ignazio Spadafora en 1876).

La présence féminine était absolument dérisoire. Si l'on observe attentivement le tableau $\mathrm{n}^{\circ} 1$, nous ne trouvons que trois noms féminins : Providenza Diliberto (1866-1868), Marie Clément (1891-1892) et Adolfina Flores (1894-1898). Les deux premières n'enseignaient que dans des écoles privées et même, en ce qui concerne la première, que dans des premières classes. La troisième a été nommée à la suite de la création de classes de jeunes filles. Or, celle-ci, tout en étant en possession de tous les titres requis, recevait un salaire inférieur à celui de son homologue masculin. Nous perdons d'autre part les traces des gagnantes aux concours locaux : Thérèse Albini 
(1876), Providenza Lucania (1878). Enfin l'établissement pour jeunes filles le plus prestigieux de Palerme, le Maria Adélaïde, a toujours eu un homme (francophone) comme enseignant (Atanase Prétrel et, ensuite, son fils Auguste).

Les hiérarchisations des salaires étaient infinies (tableau 2). Deux professeurs de catégorie identique qui enseignaient au nord ou au sud de l'Italie n'étaient pas rétribués de la même manière (il s'en fallait de beaucoup !12). Les rémunérations variaient même d'une commune à l'autre en fonction du nombre des habitants. L'Etat était aussi moins généreux que les mairies comme nous le démontre cette lettre du directeur de l'Ecole technique Gaggini qui signalait le fait que l'enseignante de français d'une classe dédoublée ${ }^{13}$ de son école ne touchait que $23 £$ (de l'Etat) alors que la concierge, prise en charge par la municipalité, en gagnait presque le double $(45 £)^{14}$. D'autre part, comme nous l'avons déjà vu, une femme, à titre égal, recevait moins qu'un homme et, comble de l'absurde, même l'indemnité de transport était inférieure ${ }^{15}$. Par ailleurs, les enseignants des lycées, des classes supérieures, des matières " nobles " (italien, mathématiques, ou matières fondamentales des écoles et instituts techniques ${ }^{16}$, etc.) percevaient un traitement supérieur à celui des autres. Les salaires sont tellement bas que les professeurs sont obligés d'enseigner dans plusieurs écoles à la fois (tableau 1). Des rapports annuels des directeurs au ministère signalent qu'ils étaient obligés de quitter leur classe avant la fin des cours pour de toute façon arriver en retard dans l'autre école. Réunir tous les enseignants lors de conseils de classe relevait de la pure utopie. Enfin, les salaires ne furent pas réévalués pendant des décennies.

En fait, ces enseignants n'avaient même pas la possibilité de se payer les livres nécessaires et leur niveau culturel jusqu'au début du XXe siècle, comme le constate la Commissione Reale de 1905 (Bochicchio, 1993 : 24), était tout à fait insatisfaisant. Nous avons déjà signalé (Lillo, 1995 : 146) à ce propos le cas d'enseignants qui n'avaient pas les moyens d'aller à Naples (siège d'examen) pour passer la " patente ». ${ }_{4}$

En 1886, la revue "La Scuola Moderna " soulignait encore avec force ce problème ${ }^{17}$ d'autant qu'un retard intolérable des paiements entre 1860 à1865, et même après, s'ajoutait à l'insuffisance des salaires. Ainsi, en 1878, le directeur de l'Ecole technique Gaggini envoyait une lettre pour demander au ministère de solder au professeur d'anglais les salaires de l'année précédente (P. CPS 44).

Quant aux retraites, on appliqua après 1860 la loi en vigueur dans l'Etat piémontais tout en maintenant les droits acquis sous le précédent gouvernement : trente ans de cotisations ( $2,5 \%$ du salaire) pour une retraite égale au salaire, vingt ans pour la moitié. En 1862 on ne dénombrait que quatre enseignants retraités avec la totalité de leur salaire (P. Pref. AG 119). Mais les salaires ont toujours été tellement bas qu'il est fort probable que le nombre des retraités de l'Education nationale ait toujours été des plus limités, en fait les cartons d'archives intitulés "Pensioni » se réfèrent presque uniquement à des retraites militaires et, plus rarement, ecclésiastiques.

\section{Les livres (tableau 3)}

33 Les grammaires de Vincenzo Leitenitz (premier et deuxième niveaux), publiées à Naples sans interruption de 1857 à 1922, étaient les manuels les plus diffusés dans les établissements palermitains ${ }^{18}$. La raison essentielle en était le prix, inférieur par exemple à celui d'ollendorf (respectivement $£ 1,50$ et $£ 3$ contre $£ 5$ ). Le recteur de l'Académie avait aussi envoyé une lettre (1877) à toutes les écoles pour demander aux 
professeurs d'uniformiser leurs choix afin de rendre plus homogène l'enseignement, de faciliter les passages d'une école à l'autre et d'éviter aux parents des dépenses inutiles ${ }^{19}$. Les deux cours (respectivement 250 et 500 pages environ) étaient organisés de la même façon : d'abord la prononciation, sans transcription phonétique mais seulement la traduction des phrases à lire, ensuite la morphosyntaxe qui considérait les différentes règles et leurs exceptions, quelques pages sur l'orthographe, des exercices de traduction sur les différentes parties du discours, enfin une nomenclature thématique des mots les plus communs. Le Deuxième cours présentait en plus une phraséologie comparée et des proverbes. Tout était basé sur l'enseignement déductif de la grammaire et les exercices de traduction étaient seuls pratiqués (les dialogues, uniquement en italien, n'existaient qu'en vue de la traduction).

L'anthologie littéraire la plus répandue était La France littéraire ou les prosateurs et les poètes français depuis Pascal et Malherbe jusqu'à nos jours. Lectures choisies avec introduction, notices biographiques et notes explicatives de Joseph Poerio ${ }^{20}$. Elle a été publiée de 1873 jusqu'au début du XX ${ }^{e}$ siècle. Divisée en deux grandes parties : " Les classiques et le $\mathrm{XVIII}^{\mathrm{e}}$ » et " Les contemporains et l'école romantique », elle regroupait les auteurs en deux catégories : les «prosateurs » d'une part, les " poètes » d'autre part. Les auteurs privilégiés (de sept à quatorze morceaux choisis) étaient : Bossuet, Fénelon, La Fontaine, Voltaire, Jean-Jacques Rousseau (ces deux derniers étant les préférés avec 14 et 12 textes respectivement), Buffon, Bernardin de Saint-Pierre, Béranger, Hugo. Plus que sur la morale ou le patriotisme, ce manuel insistait volontiers sur les événements et les grands hommes de l'histoire (c'est, par exemple, un Voltaire surtout historien qu'il nous présentait). D'autre part, les auteurs du XIXe étaient sous-évalués par rapport à ceux des deux siècles précédents.

Malgré la diffusion progressive des morceaux choisis, quelques rares enseignants continuèrent à proposer des textes intégraux : Y Histoire naturelle de Buffon, le Discours sur l'histoire universelle de Bossuet, les Caractères de La Bruyère, prolongeant ainsi, dans les choix et dans la méthodologie, les pratiques de la période précédente.

Malgré la multiplication sur le marché libraire des outils didactiques, force est de constater un nivellement par le bas dû essentiellement aux conditions socioéconomiques des enseignants et élèves de français.

\section{Programmes}

On comprendra bien que dans de telles conditions, l'écart entre les programmes officiels et les résultats obtenus était énorme. Quels qu'aient été les objectifs des programmes de 1860, 1867, 1885 (grammaire, prononciation, étude comparative des deux langues. Voir Bochicchio, 1993 : 22--24), le bilan final était affligeant pour ne pas dire nul.

Il est intéressant de voir ce qu'un des professeurs les plus célèbres de Palerme, membre du jury de français pour les concours de 1876, 1877, 1878 (Lillo, 1995 : 154), enseignant en 1876 à l'Institut technique et au Maria-Adélaïde, proposait comme programmation pour cette même année :

Programme de 1876 - français (Maria-Adélaïde, P. CPS 19, l'original est en français) :

$1^{\mathrm{er}}$ cours. 
41 continuer à insister sur une bonne prononciation, on a continué à faire des lectures et on a étudié les parties du discours sans toucher à la syntaxe, qui exige un développement plus important de l'intelligence, et que l'on a réservé pour la troisième année ${ }^{23}$ (compte rendu au recteur).

52 Si l'on se réfère aux manuels choisis, on ne peut noter qu'un décalage entre les ambitions excessives, notamment du Deuxième cours, et les objectifs, minimums, des collègues qui basaient en grande partie leur activité sur l'enseignement de la grammaire. En fait, la répétition de la phrase «si la force de la classe le permet » et le nota bene du premier texte expriment bien le désabusement de l'enseignant quant aux résultats futurs. Ces réflexions sont confirmées par des rapports combatifs de directeurs d'école déçus qui se plaignaient du niveau extrêmement bas des élèves.

\section{Conclusion}

Les conditions de travail des collègues du siècle dernier sont impensables aujourd'hui. Ils opéraient dans des conditions de survie et les préoccupations d'ordre didactique 
étaient certainement moins prioritaires que celles d'arriver à donner à manger à toute la famille.

On a pu noter un décalage important entre programmes ou instructions officiels et la pratique quotidienne révélée dans toute sa crudité par les Archives. L'étude du contenu d'un manuel ne peut pas nous permettre de présumer des résultats obtenus et tout est forcément relativisé.

La hiérarchisation excessive voulue par le gouvernement pour maintenir à tous les niveaux l'échelle sociale existante commence, en fin de siècle, à être lentement mais sûrement sapée. De façon surprenante, comme le montrent de nombreux documents, ce sont les proviseurs qui, dans le milieu de l'éducation, se font alors porteurs de solutions démocratiques et égalitaires.

\section{BIBLIOGRAPHY}

восніссніо, Franco (1993), «L'enseignement de la langue française et le système scolaire italien de 1860 à 1913 ", Documents pour l'histoire du français langue étrangère ou seconde, Paris, SIHFLES, $\mathrm{n}^{\circ} 12,16-24$.

CANCILA, Orazio (1988), Palermo, Laterza, Bari.

costA, Armando (1990), La scuola e la grande scala, Sellerio, Palerme.

GHIOTTI, Candido, Dogliani, Giustino, La lingua francese nelle scuole e le famiglie, Année IX, $\mathrm{n}^{\circ} 1$, 15 octobre 1891.

GIORDANo, Clotilde (1873), Del R. Istituto Margherita in Palermo. Cenno di Clotilde Giordano. Palerme, Lao.

GIORDANO, Clotilde (1876), VI settembre MDCCCLXXVI. Per la premiazione nel R. Istituto Margherita. Parole délia Direttrice, Palerme, Stamperia S. Bizzarrilli.

La Scuola Moderna, Palerme, $\mathrm{l}^{\mathrm{re}}$ et $2^{\mathrm{e}}$ années, 1886, 1887.

LILLO, Jacqueline (1992), « Enseignants et élèves de français à Palerme de 1800 à 1860», Documents pour l'histoire du français langue étrangère ou seconde, « Maîtres et élèves », Paris, SIHFLES, ${ }^{\circ}$ 9, 10-17.

LILLO, Jacqueline (1993), « Un avis de concours pour un poste d'enseignant de français et d'allemand à l'Institut Technique de Palerme en 1865 », Documents pour l'histoire du français langue étrangère ou seconde, Paris, SIHFLES, $\mathrm{n}^{\circ}$ 12, 25-31.

LILLO, Jacqueline (1995), «Recrutement des professeurs de français à Palerme tout de suite après l'unification de l'Italie, 1860-1878 ", Documents pour l'histoire du français langue étrangère ou seconde, Paris, SIHFLES, $n^{\circ} 15,143-159$.

ROMANo, Sergio (1977), Histoire de l'Italie du Risorgimento à nos jours, Inédit Histoire, Seuil. SANSONE, Alfonso, Storia del R. Istituto Nautico Gioeni-Trabia, 1789-1892. 
Statuto organico délia Libéra Società degl'Insegnanti in Palermo (1866), Palerme, Amenta.

\section{Sources manuscrites aux Archives Nationales}

Consiglio Provinciale Scolastico (Archives d'Etat, Palerme). (Abrégé dans le texte et les tableaux :

P. CPS) 2, 3, 6, 7, 19, 21, 44, 46.

Prefettura. Affari Generali (Archives d'Etat, Palerme). (P. Pref. AG) 115, 118, 119, 195.

Ministero Pubblica Istruzione. Consiglio Superiore. Atti 1849-1896. Istruzione Secondaria. Actes I Série (Archives d'Etat, Rome). (R. CSIS1) 21, 25, 28, 29, 31, 32, 33, 34, 103, 320.

Ministero Pubblica Istruzione. Consiglio Superiore. Atti 1897-1904. Istruzione Secondaria. Actes II Série (Archives d'Etat, Rome). (R. CSIS2) 101.

Ministero Pubblica Istruzione. Consiglio Superiore 1849-1903. Atti Versati Posteriormente (Archives d'Etat, Rome). (R. CS) 2, 5, 8, 10.

Ministero Pubblica Istruzione. Divisione Scuole Medie 1860-1896. 1) Licei e Ginnasi (Archives d'Etat, Rome). (R. SMLG) 161.

Ministero Pubblica Istruzione. Divisione Scuole Medie 1860-1896. 2) Istituti Tecnici e Nautici (Archives d'Etat, Rome). (R. SMITN) 9, 15, 39.

Ministero Pubblica Istruzione. Divisione Scuole Medie 1860-1896. 3) Scuole Tecniche (Archives d'Etat, Rome). (R. SMST) 35, 55, 57, 8

\section{APPENDIXES}

\section{Tableau $\mathbf{n}^{\circ} 1$}

\begin{tabular}{|c|c|c|c|c|}
\hline Année sc. & Institution & Prof. de français & Diplôme & Source \\
\hline (1860)-1861 & Lycée national & Antonio Macaluso ${ }^{24}$ & & P. CPS 27 R. \\
\hline & & Auguste Forget & & CSISI 31 \\
\hline $1861-1862$ & Gymnase national & $\begin{array}{l}\text { Antonio di Marzo } \\
\text { (nomination annuelle } \\
\text { provisoire) }\end{array}$ & & R. CSISI 28 \\
\hline $1861-1862$ & Ecole technique & $\begin{array}{l}\text { Enrico Zerilli } \\
\text { (nomination annuelle } \\
\text { provisoire) }\end{array}$ & & R. CSISI 31 \\
\hline $1862-1863$ & $\begin{array}{l}\text { Institut technique d'état } \\
\text { («Governativo ») }\end{array}$ & $\begin{array}{l}\text { Salvatore Milano (anglais } \\
\text { et français) }\end{array}$ & & R. SMITN 40 \\
\hline $1864-1868$ & Institut R. Marine marchande & Pietro Sebogondio ${ }^{25}$ & Pat. & $\begin{array}{l}\text { P.Pref.196 } \\
\text { R.SMITN } 39\end{array}$ \\
\hline $1866-1868$ & Institut Italiano (filles) ${ }^{26}$ & $\begin{array}{l}\text { Provvidenza Diliberto (1e } \\
\text { année) }\end{array}$ & & \\
\hline
\end{tabular}




\begin{tabular}{|c|c|c|c|c|}
\hline $1867-1873$ & Institut R. Margherita (filles) & Augusto Coussol & & P. CPS 21 \\
\hline $1869-1870$ & Institut R. Marine marchande & $\begin{array}{l}\text { Milano (français et } \\
\text { anglais) }\end{array}$ & & R. SMITN 40 \\
\hline $1869-1875$ & Epicarno (privé) & Francesco Controtti & & P. CPS 21 \\
\hline $1869-1870$ & Collège San Rocco & $\begin{array}{l}\text { Carlo Dosswald (classes } \\
\text { sip.) }\end{array}$ & Pat. & P. CPS 8 \\
\hline $1870-1881^{27}$ & Institut R. Marine marchande & Auguste Coussol & & R. MITN 40 \\
\hline $1871-1877$ & Institut Marie-Adélaïde & Atanasio Prétrl & & P.CPS 19 \\
\hline $1872-1873$ & $\begin{array}{l}\text { Institut littéraire Randazzo } \\
\text { (privé) }\end{array}$ & Augusto Coussol & & P. CPS 21 \\
\hline 1873 & Ecole technique $\mathrm{R}^{28}$ & Federico Carisi & Pat. ${ }^{29}$ & P. CPS 34 \\
\hline $1873-1878$ & Ecole technique R. Piazzi & $\begin{array}{l}\text { Francesco Milano } \\
\text { Reggente 2e classe }\end{array}$ & (Pat.) & $\begin{array}{l}\text { P. CPS } 34 \text { P. } \\
\text { CPS } 44\end{array}$ \\
\hline $187 ?$ & $\begin{array}{l}\text { Collège R. de musique «Bon } \\
\text { Pasteur » }\end{array}$ & Frencesco Milani & Pat. & P. CPS 2 \\
\hline $1874-1877$ & $\begin{array}{l}\text { Ecole technique municipale } \\
\text { "pareggiata" (reconnu) }\end{array}$ & Giuseppe Stabile & Pat. & P. CPS 34 \\
\hline $1874-1877$ & $\begin{array}{l}\text { Institut littéraire Randazzo } \\
\text { (privé) }\end{array}$ & Giuseppe Stabile & Pat. & P. CPS 27 \\
\hline avant 1877 & Ecole technique R. principale & Enrico Ruffo ${ }^{30}$ & Pat. & $\begin{array}{l}\text { P. CPS } 3 \text { P. } \\
\text { CPS } 27\end{array}$ \\
\hline 1879 & & & & \\
\hline $1874-1876$ & $\begin{array}{l}\text { Ecole technique R.bis } \\
\text { (publique) }^{31}\end{array}$ & Francesco Milano ${ }^{32}$ & (Pat. ?) & $\begin{array}{l}\text { P.CPS } 5 \text { P.CPS } \\
21\end{array}$ \\
\hline $1874-1876$ & Collège San Rocco & Enrico Ruffo Barbalonga & $\begin{array}{l}\text { Pat. } \\
\text { (sup) }\end{array}$ & P.CPS 8 \\
\hline $1875-1876$ & Institut Galilée (privé) & Raffaello Spina & & P.CPS 27 \\
\hline $1875-1878$ & Collège San Rocco & Antonino Macaluso ${ }^{33}$ & Pat. & P.CPS 8 \\
\hline $1876-1878$ & Ecole technique R. Gaggini & $\begin{array}{l}\text { Enrico Ruffo Barbalonga } \\
\text { (suppléant) }\end{array}$ & & \\
\hline $1876-1877$ & Institut technique R ; & Atanasio Prétrel & & P. CPS 16 \\
\hline
\end{tabular}




\begin{tabular}{|c|c|c|c|c|}
\hline $1876-1877$ & $\begin{array}{l}\text { Ecole technique municipale } \\
\text { pareggiata (reconnue) }\end{array}$ & Giuseppe Stabile & & P. CPS 27 \\
\hline $1876-1879$ & Maria Adélaïde & Atanasio Prétrel & & $\begin{array}{l}\text { P. CPS } 19 \text { R. } \\
\text { SMLG } 161\end{array}$ \\
\hline $1876-1877$ & $\begin{array}{l}\text { Ecole technique privée } \\
\text { «Galileo» }\end{array}$ & Raffaello Spina & Pat. & P. CPS 27 \\
\hline $1876 \ldots$ & Chez lui (cours privés) & Carlo Dosswald ${ }^{34}$ & Pat. & P. CPS 31 \\
\hline $1877-1878$ & Ecole technique R. Gaggini & $\begin{array}{l}\text { (Prof. Enrico Ruffo) Pietro } \\
\text { Grossetti }\end{array}$ & Pat. & P. CPS 34 \\
\hline $1877-1878$ & \multicolumn{2}{|c|}{ Collège de S. Marie La Charité Carlo Dosswald } & Pat. & P.CPS6 \\
\hline 1877 & Epicarmo (privé) & $\begin{array}{l}\text { Salvatore Somma (Ie } \\
\text { année tech.) }\end{array}$ & Pat & P. CPS 25 \\
\hline $1877-1878$ & Ecole du soir municipale & Léon Des Pierres & & P. CPS 16 \\
\hline $1877-1878$ & Collège de Marie Giusino & Carmelo Blanca (prêtre) & & P. CPS 6 \\
\hline $1877-1878$ & Ecole technique R. Piazzi & Enrico Ruffo & & P CPS 44 \\
\hline $1877-78 ?$ & Collège de Marie au Carmel & Carlo Dosswald & Pat. & P. CPS 8 \\
\hline $1877, .$. & Leçons particulières de maths & Antonio Iacona ${ }^{35}$ & & P CPS 32 \\
\hline & et de français & & & \\
\hline $1879-1880$ & Institut technique R. & Atanasio Prétrel & & R. SMLG 161 \\
\hline $1879-1880$ & Institut R. Marie-Adélaïde & AtanasioPrétrel & & R. SMLG 161 \\
\hline $1879-1880$ & Ecole technique R. Piazzi & Enrico Ruffo & Pat. & R. SMLG 161 \\
\hline $1880-1881$ & Institut technique R. (public) & Ignazio Mastropasqua & Pat.? & $\begin{array}{l}\text { Per. est. o } \\
\text { sopr. } n^{\circ} 281\end{array}$ \\
\hline & Lycée Umberto (public) ${ }^{36}$ & & & \\
\hline $1881-1882$ & Ecole technique R. Piazzi & Enrico Ruffo & & R.SMST35 \\
\hline $1883-1885$ & Ecole technique R. Scinà & Giuseppe Stabile & Pat? & R. SMST35 \\
\hline $1883-1884$ & Institut technique Parlatore & Ramat & & R. SMITN9 \\
\hline $1884-1885$ & Institut technique Parlatore & $\begin{array}{l}\text { Cacciardi (R) Icilio } \\
\text { Bogliani }\end{array}$ & & R. SMITN9 \\
\hline $1885-1886$ & Ecole technique R. Scinà & Giuseppe Stabile & Pat $\lll$ & R. SMST35 \\
\hline
\end{tabular}




\begin{tabular}{|c|c|c|c|c|}
\hline $1886-1887$ & Ecole technique R. Gaggini & Francesco Milano & & R. SMST35 \\
\hline $1886-1887$ & Ecole technique R. d'Acquisto & Nicolô Agalbato & & R. SMST35 \\
\hline $1886-1887$ & Ecole technique R. Piazzi & Enrico Alessandrini & & R.SMST35 \\
\hline $1886-1887$ & Ecole technique R. Scinà & Giuseppe Stabile & Pat. & R.SMST35 \\
\hline 1886-1891 & Institut technique R. & $\begin{array}{l}\text { Edoardo Cacciardi Icilio } \\
\text { Bogliani }\end{array}$ & & R. SMITN 40 \\
\hline $1891-1892$ & Ecole technique R. Gaggini & 3 ! prof & & R.SMST35 \\
\hline $1891-1892$ & $\begin{array}{l}\text { Collège de Marie Giusino } \\
\text { (privé) }\end{array}$ & Marie Clément ${ }^{37}$ & Pat. & $\begin{array}{l}\text { P. Pref. AG } \\
706\end{array}$ \\
\hline $1892-1893$ & Ecole technique R. d'Acquisto & Nicolo Agalbato & Abil. & R. SMST 35 \\
\hline $1892-1895$ & Ecole technique R. Scinà & Giuseppe Atabile & Pat. & $\begin{array}{l}\text { R. SMST } 35 \text { R. } \\
\text { SMST } 57\end{array}$ \\
\hline & & $\begin{array}{l}\text { Stanislas Chargé (classes } \\
\text { ajoutées }\end{array}$ & Pat. & \\
\hline 1894-1895 & Ecole technique R. Gaggini & Ferninando Pioti & & R. SMST 57 \\
\hline & & $\begin{array}{l}\text { Adolfina Flores (classes } \\
\text { j.f.) }\end{array}$ & Pat. & \\
\hline $1894-1898$ & Ecole technique R. Gaggini & $\begin{array}{l}\text { Adolfina Flores (classes } \\
\text { j.f.) }\end{array}$ & Pat. & R. SCIS2 101 \\
\hline 1895-1896 & Ecole technique R. d'Acquisto & $\begin{array}{l}\text { Carlo Doswald (suppléant } \\
\text { 1e ennée sections B et C) }\end{array}$ & Pat. & R. SMST 35 \\
\hline 1896-1897 & Ecole technique R. Gaggini & $\begin{array}{l}\text { Simoncini Scaglione } \\
\text { Giovanni }\end{array}$ & & R. CSIS2 \\
\hline $1896-1897$ & Ecole technique R. Scinà & Farina Giuseppe & & R. CSIS2 101 \\
\hline
\end{tabular}

Abil. : abilitazione

Pat. : patente

R: Royal /Royale

Per. est. o sopr. : Bibliothèque régionale de Palerme. «Periodici estinti o soppressi ».

$\mathrm{P}+$ dénomination des cartons : Archives d'Etat à Palerme.

$\mathrm{R}+$ dénomination des cartons : Archives d'Etat à Rome. 
P. CPS : Archives d'Etat à Palerme, Consiglio Provinciale Scolastico.

P. Pref. AG : Archives d'Etat à Palerme, Prefettura, Affari Generali.

R. CSIS1 : Archives d'Etat à Rome, Ministero Pubblica Istruzione. Consiglio Superiore. Atti 1849-1896. Istruzione Secondaria. Actes I Série.

R. CSIS2 : Archives d'Etat à Rome, Ministero Pubblica Istruzione. Consiglio Superiore. Atti 1897-1904. Istruzione Secondaria. Actes II Série.

R. CS : Archives d'Etat à Rome, Ministero Pubblica Istruzione. Consiglio Superiore 1849-1903. Atti Versati Posteriormente.

R. SMLG : Archives d'Etat à Rome, Ministero Pubblica Istruzione. Divisione Scuole Medie 1860-1896. 1) Licei e Ginnasi.

R. SMITN : Archives d'Etat à Rome, Ministero Pubblica Istruzione. Divisione Scuole Medie 1860-1896. 2) Istituti Tecnici e Nautici.

R. SMST: Archives d'Etat à Rome, Ministero Pubblica Istruzione. Divisione Scuole Medie 1860-1896.3) Scuole Tecniche.

Tableau $\mathrm{N}^{\circ} 2$

Salaires annuels

\begin{tabular}{|c|c|c|c|c|c|}
\hline Année & Ecole & \begin{tabular}{|l} 
Prof; \\
Français
\end{tabular} & $\begin{array}{l}\text { Prof. lettres } \\
\text { ou math. }\end{array}$ & $\begin{array}{l}\text { Heures/ } \\
\text { semaine }\end{array}$ & Sources \\
\hline \multirow[t]{2}{*}{$(1860)-1861$} & Lycée national Forget ${ }^{38}$ & $£ 600$ & & $\begin{array}{l}\text { «3 fois tous } \\
\text { les j.» }\end{array}$ & $\begin{array}{l}\text { R. CSISI } \\
31\end{array}$ \\
\hline & Macaluso & $£ 750$ & & & \\
\hline $1861-1862$ & Collège nautique ${ }^{39}$ & $£ 919$ & $£ 1225,50$ & & Sanson \\
\hline $1861-1862$ & $\begin{array}{l}\text { Gymnase national } \\
\text { Antonio di Marzo }\end{array}$ & $£ 600$ & $£ 1000$ & $\begin{array}{l}3 \text { cours/ } \\
\text { classe III }\end{array}$ & $\begin{array}{l}\text { R. CSISI } \\
28\end{array}$ \\
\hline \multirow[t]{2}{*}{$1861-1862$} & Ecole technique & $£ 1120$ & $£ 1600$ & & $\begin{array}{l}\text { R. CSISI } \\
31\end{array}$ \\
\hline & Zerilli Enrico & 《 Incaricato» & & & $\begin{array}{l}\text { R. CSISI } \\
25\end{array}$ \\
\hline $1863-1864$ & Collège San Rocco & $£ 229,50$ & $£ 459$ (4 cl.) & & $\begin{array}{l}\text { P. Pref } \\
\text { AG195 }\end{array}$ \\
\hline \multirow[t]{2}{*}{$1864-1865$} & $\begin{array}{l}\text { Institut R. de Marine } \\
\text { marchande }\end{array}$ & $£ 1600$ & & & $\begin{array}{l}\text { R. SMITN } \\
39\end{array}$ \\
\hline & Pietro Sebergondio & \begin{tabular}{|l} 
“Incaricato" \\
$£ 1600$ (sic) \\
« Reggente»
\end{tabular} & & & \\
\hline
\end{tabular}




\begin{tabular}{|c|c|c|c|c|c|}
\hline $1868-1869$ & $\begin{array}{l}\text { Institut R; industriel et } \\
\text { professionnel (De } \\
\text { Marchi) }\end{array}$ & $\begin{array}{l}£ 1200 \\
\text { «Incaricato » }\end{array}$ & & & $\begin{array}{l}\text { R. SMITN } \\
39\end{array}$ \\
\hline $187 ?$ & $\begin{array}{l}\text { Collège de musique "Bon } \\
\text { Pasteur" } \\
\text { Francesco Milani }\end{array}$ & $£ 250$ & & 6 & P. CPS 2 \\
\hline $1870-1871$ & $\begin{array}{l}\text { Institut R. Marine } \\
\text { marchande } \\
\text { Auguste Coussol }\end{array}$ & $£ 1200^{40}$ & & & \\
\hline $1876-1878$ & $\begin{array}{l}\text { Marie-Adélaïde } \\
\text { Atansio Prétrel }\end{array}$ & $£ 900$ & & $71 / 2$ & P. CPS 8 \\
\hline $1877-1878$ & $\begin{array}{l}\text { Collège S. Marie La } \\
\text { Charité } \\
\text { Carlo Dosswald }\end{array}$ & $£ 306$ & & 6 & \\
\hline $1877-1878$ & $\begin{array}{l}\text { Institut technique R. } \\
\text { Atanasio Prétrel }\end{array}$ & $£ 1200$ & $£ 2640$ & & \\
\hline $1880-1881$ & $\begin{array}{l}\text { Ecole technique R. Piazzi } \\
\text { Enrico Ruffo }\end{array}$ & $£ 1920$ & & & $\begin{array}{l}\text { R.SMST } \\
35\end{array}$ \\
\hline $1883-1885$ & $\begin{array}{l}\text { Ecole technique R. } \\
\text { Scienà Giuseppe Stabile }\end{array}$ & $£ 2160$ & & & $\begin{array}{l}\text { R.SMST } \\
35\end{array}$ \\
\hline $1885-1886$ & $\begin{array}{l}\text { Ecole technique R. Piazzi } \\
\text { Loretz }\end{array}$ & & & & $\begin{array}{l}\text { R.SMST } \\
35\end{array}$ \\
\hline $1886-1887$ & $\begin{array}{l}\text { Ecole technique R. } \\
\text { d'Acquisto Nicolo } \\
\text { Agalbatp }\end{array}$ & $£ 1728$ & 1920 & & $\begin{array}{l}\text { R.SMST } \\
35\end{array}$ \\
\hline $1886-1887$ & $\begin{array}{l}\text { Ecole technique } \\
\text { R.Gaggini } \\
\text { Francesco Milano } \\
\text { (consirlé pour } 3 \text { ans) }\end{array}$ & $£ 1728$ & 1920 & & $\begin{array}{l}\text { R.SMST } \\
35\end{array}$ \\
\hline $1886-1887$ & Ecole technique R. Piazzi & $£ 1728$ & 2640 & & $\begin{array}{l}\text { R.SMST } \\
35\end{array}$ \\
\hline & Enrico Alesandrini & & Titulaire & & \\
\hline $1886-1887$ & Ecole technique R. Scinà & $£ 2400$ & 2640 & & $\begin{array}{l}\text { R.SMST } \\
35\end{array}$ \\
\hline & Giuseppe Stabile & Titulaire & Titulaire & & \\
\hline
\end{tabular}




\begin{tabular}{|c|c|c|c|c|c|}
\hline $1886-1887$ & Institut technique R. & $£ 2160$ & & 9 & $\begin{array}{l}\text { R. SMITN } \\
40\end{array}$ \\
\hline & Edoardo Cacciardi & Titulaire & & & \\
\hline & Icilio Bogliani & $\begin{array}{l}£ 2112 \\
\text { « Reggente » }\end{array}$ & & & \\
\hline $1891-1892$ & $\begin{array}{l}\text { Collège Marie de Giusino } \\
\text { Marie Clément }\end{array}$ & $£ 420$ & $\begin{array}{l}£ 900 \text { (corso } \\
\text { perfettivo) }\end{array}$ & & \\
\hline $1892-1893$ & $\begin{array}{l}\text { Ecole technique R. } \\
\text { d'Acquisto }\end{array}$ & $£ 1920$ & $£ 2112$ & & $\begin{array}{l}\text { R. SMST } \\
35\end{array}$ \\
\hline & Nicolo Agalbato & Titulaire & Titulaire & & \\
\hline $1892-1893$ & $\begin{array}{l}\text { Ecole technique R. } \\
\text { Gaggini } \\
\text { classe de jeunes filles } \\
\text { (classes ajoutées) }\end{array}$ & $\begin{array}{l}£ 23 \text { par } \\
\text { mois ! }{ }^{41}\end{array}$ & & & \\
\hline $\begin{array}{l}1896(10 / 4 \text { au } \\
31 / 7)\end{array}$ & $\begin{array}{l}\text { Ernest Coussol } \\
\text { (suppléant) }\end{array}$ & $\begin{array}{l}£ 100 \text { par } \\
\text { mois }\end{array}$ & & & $\begin{array}{l}\text { R. SMITN } \\
40\end{array}$ \\
\hline $1897-1898$ & Ecole technique R. de J.F. & $\begin{array}{l}£ 100 \text { par } \\
\text { mois }\end{array}$ & & & $\begin{array}{l}\text { R. CSIS2 } \\
101\end{array}$ \\
\hline & Adolfina Flores & 《 Incaricata » & & & \\
\hline $1897-1898$ & $\begin{array}{l}\text { Ecole technique R. Scinà } \\
\text { (classes ajoutées) }\end{array}$ & $£ 1536$ & & & $\begin{array}{l}\text { R. CSIS2 } \\
101\end{array}$ \\
\hline $1897-1898$ & $\begin{array}{l}\text { Institut R. Marine } \\
\text { marchande } \\
\text { Constantino Lo Cascio }\end{array}$ & $£ 2160$ & & 8 & $\begin{array}{l}\text { R. CSIS2 } \\
101\end{array}$ \\
\hline
\end{tabular}

\begin{tabular}{|l|l|l|l|l|l|}
\hline A. sc. & Institution & Classes & Manuels de français & Auteur & Sources \\
\hline $\begin{array}{l}\text { 1860-1870 } \\
\text { (environ) }\end{array}$ & $\begin{array}{l}\text { Institut privé } \\
\text { Epicarno }\end{array}$ & $?$ & Grammaire $1^{\text {er }}$ niveau & $\begin{array}{l}\text { Vincenzo } \\
\text { Leitenitz }\end{array}$ & P. CPS 21 \\
\hline & & & Letture & $\begin{array}{l}\text { Vincenzo } \\
\text { Leitenitz }\end{array}$ & \\
\hline $1868-1870$ & $\begin{array}{l}\text { Institut R. de } \\
\text { Marine } \\
\text { marchande }\end{array}$ & 1.2. & $1^{\text {er }}$ et $2^{\text {e } \text { niveaux (corsi) }}$ & Vincenzo & Reitenitz \\
RMITN \\
39. SMITN \\
40
\end{tabular}




\begin{tabular}{|c|c|c|c|c|c|}
\hline $1874-1875$ & $\begin{array}{l}\text { Ecole technique R. } \\
\text { bis }\end{array}$ & $?$ & Prime letture & $\begin{array}{l}\text { Vincenzo } \\
\text { Leitenitz }\end{array}$ & P. CPS 21 \\
\hline & & & Grammaire $2^{\mathrm{e}}$ niveau & $\begin{array}{l}\text { Vincenzo } \\
\text { Leitenitz }\end{array}$ & \\
\hline \multirow[t]{2}{*}{$1874-1875$} & $\begin{array}{l}\text { Ecole technique R. } \\
\text { bis }\end{array}$ & $?$ & Grammaire & $\begin{array}{l}\text { Vincenzo } \\
\text { Leitenitz }\end{array}$ & P. CPS 21 \\
\hline & & & Letture francesi & Brianzi & \\
\hline \multirow[t]{2}{*}{$1874-1875$} & $\begin{array}{l}\text { Ecole technique R. } \\
\text { bis }\end{array}$ & ? & Grammaire $2^{\mathrm{e}}$ niveau & $\begin{array}{l}\text { Vincenzo } \\
\text { Leitenitz }\end{array}$ & P. CPS 21 \\
\hline & & & Letture francesi & Poerio & \\
\hline $1876-1877$ & Marie-Adélaïde & 1 & $\begin{array}{l}\text { Petite Grammaire } 1^{\mathrm{er}} \\
\text { niveau }\end{array}$ & $\begin{array}{l}\text { Vincenzo } \\
\text { Leitenitz }\end{array}$ & P. CPS 19 \\
\hline $1876-1877$ & Marie-Adélaïde & 2 & Petite Grammaire $2^{\mathrm{e}}$ niveau & $\begin{array}{l}\text { Vincenzo } \\
\text { Leitenitz }\end{array}$ & P. CPS 19 \\
\hline $1876-1878$ & Marie-Adélaïde & $\mathrm{V}$ & Grammaire $1^{\mathrm{er}}$ niveau & $\begin{array}{l}\text { Vincenzo } \\
\text { Leitenitz }\end{array}$ & P. CPS 19 \\
\hline \multirow[t]{2}{*}{$1876-1878$} & Marie-Adélaïde & VI & Grammaire $2^{\mathrm{e}}$ niveau & $\begin{array}{l}\text { Vincenzo } \\
\text { Leitenitz }\end{array}$ & P. CPS 19 \\
\hline & & & Exercices de mémoire & G. Belèze & \\
\hline \multirow[t]{2}{*}{$1876-1878$} & Marie-Adélaïde & VII & Grammaire $2^{\mathrm{e}}$ niveau & $\begin{array}{l}\text { Vincenzo } \\
\text { Leitenitz }\end{array}$ & P. CPS 19 \\
\hline & & & Exercices de mémoire & G. Belèze & \\
\hline $1876-1877$ & $\begin{array}{l}\text { Ecole technique } \\
\text { municipale }\end{array}$ & 1 & Grammaire $1^{\mathrm{er}}$ niveau ${ }^{42}$ & $\begin{array}{l}\text { Vincenzo } \\
\text { Leitenitz }\end{array}$ & P. CPS 34 \\
\hline $1876-1877$ & $\begin{array}{l}\text { Ecole technique } \\
\text { municipale }\end{array}$ & 2 & Grammaire $2^{\mathrm{e}}$ niveau ${ }^{43}$ & $\begin{array}{l}\text { Vincenzo } \\
\text { Leitenitz }\end{array}$ & P. CPS 34 \\
\hline \multirow[t]{2}{*}{$1876-1877$} & $\begin{array}{l}\text { Ecole technique } \\
\text { municipale }\end{array}$ & 3 & Grammaire $2^{\mathrm{e}}$ niveau & $\begin{array}{l}\text { Vincenzo } \\
\text { Leitenitz }\end{array}$ & P. CPS 34 \\
\hline & & & Letture connesse $(?)^{44}$ & $\begin{array}{l}\text { Vincenzo } \\
\text { Leitenitz }\end{array}$ & \\
\hline \multirow[t]{2}{*}{$1876-1877$} & $\begin{array}{l}\text { Ecole technique R. } \\
\text { principale }\end{array}$ & 1 & Grammatica francese & Ollendorff ${ }^{45}$ & R. CS 10 \\
\hline & & & Première lectures françaises & Leitenitz & \\
\hline
\end{tabular}




\begin{tabular}{|c|c|c|c|c|c|}
\hline $1876-1877$ & $\begin{array}{l}\text { Ecole technique } \mathrm{R} . \\
\text { principale }\end{array}$ & 2 & $\begin{array}{l}\text { Grammatica francese } \\
\text { 2e niveau }\end{array}$ & Ollendorff & R. CS 10 \\
\hline $1876-1877$ & $\begin{array}{l}\text { Ecole technique } \mathrm{R} . \\
\text { principale }\end{array}$ & 3 & $\begin{array}{l}\text { Grammatica francese } \\
\text { 2e niveau }\end{array}$ & Leitenitz (sic) & R. CS 10 \\
\hline $1876-1877$ & $\begin{array}{l}\text { Ecole technique R. } \\
\text { d'Acquisto }\end{array}$ & $1 \& 2$ & $\begin{array}{l}\text { Grammatichetta ad uso } \\
\text { delle scuole tecniche }\end{array}$ & $\begin{array}{l}\text { Leitenitz } \\
\text { Naples, } 1876\end{array}$ & R. CS 10 \\
\hline \multirow[t]{2}{*}{$1876-1877$} & $\begin{array}{l}\text { Ecole technique R. } \\
\text { d'Acquisto }\end{array}$ & 3 & Grammatica & $\begin{array}{l}\text { Leitenitz } \\
\text { Naples, } 1876\end{array}$ & R. CS 10 \\
\hline & & & Secondes Lectures & $\begin{array}{l}\text { Leitenitz } \\
\text { Naples, } 1876\end{array}$ & \\
\hline $1877-1878$ & $\begin{array}{l}\text { Ecole technique R. } \\
\text { Gaggini } 1\end{array}$ & 1 & Grammaire $1^{e r}$ niveau & $\begin{array}{l}\text { Vincenzo } \\
\text { Leitenitz }\end{array}$ & $\begin{array}{l}\text { P. CPS } \\
7 / 16\end{array}$ \\
\hline $1877-1878$ & $\begin{array}{l}\text { Ecole technique R. } \\
\text { Gaggini } 2\end{array}$ & 2 & Grammaire $1^{e r}$ niveau & $\begin{array}{l}\text { Vincenzo } \\
\text { Leitenitz }\end{array}$ & $\begin{array}{l}\text { P. CPS } \\
7 / 16\end{array}$ \\
\hline \multirow[t]{2}{*}{$1877-1878$} & $\begin{array}{l}\text { Ecole technique R. } \\
\text { Gaggini } 3\end{array}$ & 3 & Grammaire $2^{e}$ niveau & $\begin{array}{l}\text { Vincenzo } \\
\text { Leitenitz }\end{array}$ & P. CPS 7 \\
\hline & & & La Francia letteraria & Poerio & \\
\hline $1876-1878$ & $\begin{array}{l}\text { Ecole technique R. } \\
\text { Piazzi }\end{array}$ & 1 & Grammaire $1^{e r}$ niveau & $\begin{array}{l}\text { Vincenzo } \\
\text { Leitenitz } \\
\text { Naples } 1875\end{array}$ & $\begin{array}{l}\text { P. } \\
\text { CPS7R.CS } \\
10\end{array}$ \\
\hline $1876-1878$ & $\begin{array}{l}\text { Ecole technique R. } \\
\text { Piazzi }\end{array}$ & 2 & Grammaire $1^{e r}$ niveau & $\begin{array}{l}\text { Vincenzo } \\
\text { Leitenitz } \\
\text { Naples } 1875\end{array}$ & $\begin{array}{l}\text { P. } \\
\text { CPS7R.CS } \\
10\end{array}$ \\
\hline \multirow[t]{2}{*}{$1876-1878$} & $\begin{array}{l}\text { Ecole technique R. } \\
\text { Piazzi }\end{array}$ & 3 & Grammaire $2^{e}$ niveau & $\begin{array}{l}\text { Vincenzo } \\
\text { Leitenitz }\end{array}$ & P. CPS 7 \\
\hline & & & La Francia litteraria & $\begin{array}{l}\text { Poerio } \\
\text { Turin Paravia }\end{array}$ & \\
\hline $1877-1878$ & $\begin{array}{l}\text { Ecole technique R. } \\
\text { d'Acquisto }\end{array}$ & 1 & Grammaire $1^{e r}$ niveau & $\begin{array}{l}\text { Vincenzo } \\
\text { Leitenitz }\end{array}$ & P. CPS 7 \\
\hline $1877-1878$ & $\begin{array}{l}\text { Ecole technique R. } \\
\text { d'Acquisto }\end{array}$ & 2 & Grammaire $1^{e r}$ niveau & $\begin{array}{l}\text { Vincenzo } \\
\text { Leitenitz }\end{array}$ & P. CPS 7 \\
\hline $1877-1878$ & $\begin{array}{l}\text { Ecole technique R. } \\
\text { d'Acquisto }\end{array}$ & 3 & Grammaire $2^{e}$ niveau & $\begin{array}{l}\text { Vincenzo } \\
\text { Leitenitz }\end{array}$ & P. CPS 7 \\
\hline
\end{tabular}




\begin{tabular}{|l|l|l|l|l|l|}
\hline 1883-1884 & $\begin{array}{l}\text { Ecole technique } \\
\text { Scinà }\end{array}$ & 2 & Grammatichetta & Leitenitz & R. SMST 35 \\
\hline 1883-1884 & $\begin{array}{l}\text { Ecole technique } \\
\text { Scinà }\end{array}$ & 3 & Grammatica Il parle & Leitenitz & R. SMST 35 \\
\hline \hline $1892-1893$ & $\begin{array}{l}\text { Ecole technique R. } \\
\text { d'Acquisto }\end{array}$ & 1 & $\begin{array}{l}\text { Avviamento alla Lettura } \\
\text { francese }\end{array}$ & $?$ & Poerio \\
\hline \hline $1892-1893$ & $\begin{array}{l}\text { Ecole technique R. } \\
\text { d'Acquisto }\end{array}$ & 2 & Grammatica francese & Leitenitz & R. SMST 35 \\
\hline \hline $1892-1893$ & $\begin{array}{l}\text { Ecole technique R. SMST 35 } \\
\text { d'Acquisto }\end{array}$ & 3 & Grammatica francese & Leitenitz & R. SMST 35 \\
\hline & & & Morale pratique & Leitenitz & \\
\hline
\end{tabular}

\section{NOTES}

1. "Tutte le rendite e $i$ beni chefurono degli espulsi Gesuiti e Liguorini, eccetto quelli che... fossero espressamente destinati al culto divino, son dichiarati proprietà della Pubblica Istruzione e addetti all'uso di essa ». (« Tous les biens et revenus qui ont appartenu aux Jésuites et Rédemptoristes expulsés (du royaume), à l'exception de ceux... qui étaient uniquement utilisés pour le culte divin, sont déclarés propriété de l'Education nationale et seront mis à sa disposition »). 17 octobre 1860. Dans les intentions du gouvernement les revenus du capital devaient servir à payer les professeurs et employés de l'Education nationale.

2. Les deux premières années d'études secondaires classiques.

3. L'instruction "technique " prévoyait d'abord trois ans dans une école technique (Scuola Tecnica), et trois ou quatre ans (en fonction de la section choisie) dans un institut technique (Istituto Tecnico). Chaque année était sanctionnée par des examens finaux et la dernière année par une licenza.

4. E poiché lo scopo principale délie Scuole Tecniche è quello di avviare alla carriera commerciale, si è prestato attenzione allo studio della lingua francese tanto reclamato e dalle sociali convenienze e dal commercio. Compte rendu du directeur de la Regia Scuola tecnica bis au Provveditore, le 23 août 1875 (R CSP 21. Voir la bibliographe pour les abréviations des cartons d'archives) : « Et comme l'objectif principal des écoles techniques est de préparer à la carrière commerciale, on a donné de l'importance à l'étude de la langue française si nécessaire à la vie en société et dans le commerce 》.

5. Lettre du 26 avril 1875 du directeur de l'Institut technique au Provveditore de Palerme : « Rilengo in ogni modo che la massima parte degli alunni appartengono a famiglie di ristrettissima fortuna e che si rivolgono all 'Islituto per la sola ragione di trovare una via spedita onde procacciarsi al più presto una professione od un impiego".

6. C'est-à-dire non sicilienne.

7. Lettre du Provveditore au directeur de l'Ecole technique d'Acquisto : « è chiaro che dei molti giovani che chiedono l'iscrizione, taluni non possono vedere soddisfallo il loro desiderio » (P. CPS 46).

8. Certaines familles pouvaient en être exemptées totalement ou en partie si elles présentaient un « certificat de pauvreté». 
9. Pour l'année scolaire 1877-1878, le Collège de Marie au Capo (quartier populaire de Palerme) comptait 340 inscriptions. Au premier janvier, seulement 276 élèves fréquentaient encore les cours. Dans le courant de l'année, 20 se sont retirées pour cause de maladies et 2 pour cause de décès (P. CPS 6).

10. "Essendosi il medesimo poslo in ginocchio col capo scoperto e colla mano destra sui Sacrosanti Evangeli, alla presenza degli infrascritti testimoni,hapronunziato ad alta ed intelligibile voce la seguente formula : 'lo, ..., giuro di essere fedele al Re ed ai suoi reali successori ed osservare fedelmente lo statuto e le leggi dello stato e di esercitare le funzioni suddette nel solo scopo del bene inseparabile del Re e della Patria' ». Signature de la personne qui a juré, des témoins, du Préfet.

11. Relation du directeur de l'Ecole technique d'Acquisto au ministre (21 juillet 1896) : "E' colto, intelligente, premuroso e valente specie nel lenere severadiscipline e neifarsi rispettare e obbidire dai discenti » (« Il est cultivé, intelligent, empressé et arrive surtout à maintenir la discipline, à se faire respecter et obéir des élèves »).

12. Rapport Fileti au sujet du Collège Nautico : « Le Collège donne à peine $750 €$ par an à un professeur qui, à Gênes, en toucherait 1600 » («// Collegio corrisponde appena $€ 750$ annuali a quel professore che gode in Genova lo stipendio di $€ 1600 »)$.

13. Quand une classe était dédoublée à cause du nombre excessif d'élèves, les enseignants des nouvelles classes « ajoutées » gagnaient un salaire bien inférieur. Le niveau était presque toujours catastrophique.

14. Relation finale du directeur de la Gaggini, année scolaire 1892-1893 : «...alle brave maestre dellee classi femminili venga accordato lo stipendio di legge corne aiprofessori dellee scuole maschili e cioè perché la meschina retribuzione che è assegnata alle classi aggiunîe oltre chè è una vera ironia fiacca ogni buona volontà... nel cadente anno abbiamo dovuto subire l'umiliazione di vedere la nostra bidella pagaia dal Municipio con $€ .45$ al mese e la nostra maestra di francese retribuita dal governo con $£ 23$ ». (R. SMST 35).

15. La différence entre les sexes est telle que les maîtresses inscrites à la Libéra Società degl'Insegnanti in Palermo " peuvent assister à toutes les réunions et prendre part à toutes les discussions... mais leur vote ne sera que délibératif sur les questions scolaires et financières ». (Le signore socle maeslre potranno assistere alle adunanze e prender parte in tutte le discussioni della Società ; pero il loro voto sarà solamente deliberativo nelle quistioni scolastiche e finanziarie. Statuto organico délia Libéra Società degl'Insegnanti in Palermo, 1866.

16. "Construction de machine » par exemple.

17. Oggidi nonc'è un modesto commesso viaggiatore o non viaggiaîore che non guadagni qualche cosa di più dello stipendio di un prof essore di liceo, il quale bisogna che campi vecchio e si logori la vita fino all'ultimo per avère 8 lire al giorno di paga e 6 lire di pensioni. Peggio ancora poi è la condizione del professore di ginnasio. Corne si puo comprare libri se non ha da comprare da vestirsi e da mangiare !... Tu cominci con un tozzo di pane e con un tozzo di pane finisci. " De nos jours, il n'y a pas de commis voyageur, ou non voyageur, qui ne gagne quelque chose de plus que le salaire d'un professeur de lycée qui doit vivre vieux et se démener jusqu'au bout pour avoir huit lires de paye par jour et six lires de retraite... Comment peut-on s'acheter des livres si on n'a pas de quoi s'habiller et manger ?... On commence avec un quignon de pain et on finit avec un quignon de pain ». $\mathrm{N}^{\circ}$ 5, p. 112.

18. Vincezo Leitenitz a publié à Naples une quarantaine de Primo Corso de 1868 à 1918 environ, et une trentaine de Secondo corso de 1857 à 1922. De 1845 à 1893, il a aussi publié : Elementi di lettura francese... seguita da una raccolta di poesie..., Premières lectures. Morale pratique ou les bons exemples précédés de préceptes et de conseils..., Secondes lectures... recueil de morceaux choisis en prose et en vers... en tout une vingtaine d'éditions.

19. Tenuto présente la dichiarazione del $R$. Provveditore informato al concetto di rendere il più che sia possibile uniforme ed efficace Vistruzione che si impartisce negli Istituti sopranotati e di sgravare nei limiti del possibile i parenti dalle eccessive spese nell'acquisto dei libri di testo e di non costringerli a comprarne di nuovi nel caso di passagio degli alunni da una ad altra delle scuole (P. CPS 7). 
20. Voir à propos des anthologies littéraires les articles de Marie-Hélène Clavères et de Nadia Minerva dans ce même numéro.

21. Comme nous venons de le voir, Leitenitz est un auteur de manuels de français qui ne propose que des dialogues en italien que l'élève doit traduire en français !

22. Les mots soulignés l'ont été par Prétrel.

23. "Al primo corso si è insistito a far pronunciare per bene la lingua e afare studiare le prime quattro conjugazioni coi due ausiliari. Nel secondo poi, senza perdere di vista che l'insistenza sulla ortoepia non è mai sujficiente, si è continuato a leggere, e si sono studiate le parti del discorso senza entrare nella parte délia sintassi che esige maggior sviluppo d'intelligenza e che percio è stata assegnata al terzo corso » (P. CPS 21).

24. En 1866, Antonino Macaluso arrivera second, après Ruffo, au concours pour un poste de professeur de français dans les écoles techniques. Il obtient la patente en 1871.

25. Ex-officier en surnombre, enseigne le français et l'anglais dans ce même Institut.

26. Deviendra le Reggio Istituto Margherita. Institut " privé, provincial et communal ", il recevra du ministère des fonds pour l'école normale créée dans ses murs. Henriette Crivell y enseigne la couture. .

27. En 1881 Coussol est nommé Reggente, son salaire est augmenté de 2/10, selon les lois 30 juin et 26 décembre 1872.

28. Cette école s'appellera par la suite (1877) Regia Scuola Tecnica D'Acquisto.

29. Patente est synonyme d'abilitazione

30. A ne pas confondre avec Enrico Ruffo Barbalonga qui enseignea pendant la même période, anglais et français.

31. Deviendra la Reggia Scuola Tecnica Piazzi.

32. En 1863 Francesco Milano avait gagné un poste d'enseignant d'anglais à l'Institut technique, mais la Deputazione Provinciale veut le limoger car il a une prononciation trop américaine (Pref. 123). On suppose que le Francesco Milano cité précédemment pour l'Institut «Buon Pastore » et ce Francesco Milano sont la même personne. S'il s'agit du même enseignant, il a certainement la patente.

33. En 1866 Antonio Macaluso s'est présenté à un concours, dont faisait partie le prof. Forget, pour obtenir une place de français dans l'école technique. Il est arrivé second et n'a pas eu le poste (CPS 27).

34. Dans le journal palermitain: Il Commercio di Sicilia. Politiico-Amministratibo-TeatraleCommerciale du 9-11 novembre 1876; Année XVII n. 61 on peut lire (en italien) la petite annonce suivante: "Langues française et allemande. Le Professeur Carlo Dosswald communique qu'il habite maintenant rue Gioiamia, cour Rosano n.2, dernière étage. Depuis le début du mois il a recommencé à donner des leçons particulières de français et d'allemand chez lui, à partir de 6 heures de l'après-midi. Il fait aussi cours à des groupes. Honoraires à discuter » (CPS 31).

35. Antonio Iacona a vécu et enseigné en France.

36. Créé en 1878. Il s'agit d'un cours facultatif de français

37. Dans La lingua francese nelle scuole e nelle famiglie, année IX, $n^{\circ} 1,15$ octobre 1891 , de Ghiotti e Dogliani, on peut lire (en français) : « Résultats des examens de Brevet pour l'enseignement de la langue française donnés au mois d'avril de l'année courante dans les différentes Universités du Royaume et dans les Instituts Supérieurs... Université de Palerme, Clément Marie de Lyon, point 256/330 ». Cette année-là Marie Clément a été la seule candidate à Palerme.

38. C'est Forget qui a demandé à n'enseigner que trois jours par semaine parce que trop occupé. Il change donc son poste avec Macaluso qui prend ses heures et son salaire (R CSPI 31). Le décret $\mathrm{n}^{\circ} 306$ du 27 octobre 1860 prévoit deux professeurs de français pour le «gymnase » avec des salaires respectifs de 750 et 600 lires. La même année, dans le même établissement, un surveillant prend lui aussi 750 lires et le concierge 600 (Costa, 1990 : 506-507)!

39. Devient en 1864 le Reggio Istituto di Marine Mercantile. 
40. La moitié du salaire seulement est payée par le ministère.

41. La concierge de la même école gagne presque le double (£ 45).

42. Ce livre coûte, en $1875, £ 1,50$.

43. Ce livre coûte, en $1875, £ 3$.

44. Ce livre coûte, en $1875, £ 1,25$.

45. Ce livre est très cher : 5 lires. Il est publié à Florence. Il n'a été retrouvé dans aucune bibliothèque (Pellandra, Minerva, 1997). Les grammaires de Leitenitz, toutes publiées à Naples, sont beaucoup plus abordables.

\section{AUTHOR}

\section{JACQUELINE LILLO}

Université de Palerme 